Check for updates

Cite this: Phys. Chem. Chem. Phys., 2019, 21, 11797

Received 8th February 2019, Accepted 15th May 2019

DOI: 10.1039/c9cp00795d

rsc.li/pccp

\title{
Structural dynamics of an excited donor-acceptor complex from ultrafast polarized infrared spectroscopy, molecular dynamics simulations, and quantum chemical calculations $\uparrow+$
}

\author{
Christopher A. Rumble (D) * and Eric Vauthey (D) *
}

\begin{abstract}
The structural dynamics of an electron donor/acceptor complex (DAC) consisting of benzene and tetracyanoethylene (Bz/TCNE) solvated in $\mathrm{CH}_{2} \mathrm{Cl}_{2}$ have been investigated using ultrafast spectroscopy and mixed quantum/classical computer simulations. Population dynamics from visible and infrared transient absorption (TRIR) spectroscopy point to complex sub-10 ps dynamics followed by charge recombination on a 55-60 ps timescale. Structural dynamics involving large-scale reorganization of $\mathrm{Bz}^{\bullet+} / \mathrm{TCNE}^{\bullet-}$ radical ion pairs are revealed using TRIR anisotropy measurements. A computational study combining quantum chemical calculations and classical molecular dynamics simulations was able to reproduce the experimental electronic absorption lineshape and TRIR anisotropy dynamics, allowing for a detailed investigation of the pair conformational dynamics. Contrary to the static single structure typically assumed in descriptions of DACs, we find that neither the ground nor excited state can be described using a single, well-defined species. Instead, the pair undergoes a rearrangement from disordered $\pi$-stacks to edge-to-face $T$-shaped structures following excitation. Translational diffusion of the radical ion pairs following excitation was found to be heterogeneous and dependent on both pair separation and orientation coordinates. Given the sensitivity of charge-transfer reactions to the arrangement of donor/acceptor pairs, the structural heterogeneity and corresponding dynamics demonstrated herein must be taken into account in future modeling of charge recombination processes in DACs.
\end{abstract}

\section{Introduction}

The electronic absorption spectrum of electron donor (D) and acceptor (A) mixtures in solution is often characterized by the presence of a low-energy electronic absorption band not present in the spectra of the individual constituents. This band is due to a charge-transfer (CT) transition of a donor-acceptor complex (DAC), ${ }^{1}$ and its excitation leads to the population of an excited state that is essentially a radical ion pair (IP). ${ }^{2,3}$ Such direct optical generation of ion pairs has made DACs attractive targets for testing electron-transfer theories, as it allows one to monitor the charge-recombination (CR) dynamics of ion pairs without the complications associated with their formation upon bimolecular photoinduced electron transfer. ${ }^{3-6}$ In this respect, significant advances in our understanding of electrontransfer processes have been achieved thanks to studies of

Department of Physical Chemistry, University of Geneva, 30 Quai Ernest-Ansermet, CH-1211 Geneva 4, Switzerland. E-mail: christopher.rumble@unige.ch,

eric.vauthey@unige.ch

$\dagger$ All data are available for download at http://doi.org/10.5281/zenodo.2872150.

\# Electronic supplementary information (ESI) available. See DOI: 10.1039/ c9cp00795d
DACs, including the role of intramolecular modes $^{7-9}$ and the effect of non-equilibrium dynamics. ${ }^{10-12}$

Surprisingly, little is known about the structure of DACs in liquids, although such information is crucial for a quantitative analysis of their electron-transfer dynamics. DACs are often assumed to have a well-defined structure consisting of D and A moieties in a $\pi$-stacked face-to-face arrangement. This picture is based primarily on X-ray diffraction studies of crystalline DACs, ${ }^{13,14}$ spectroscopic measurements in supersonic jets, ${ }^{15,16}$ and quantumchemical calculations. ${ }^{17-21}$ However, a significant number of experimental observations point toward an influence of DA pair structure on DAC photophysics in liquids. Complexes with substituted benzene donors exhibit two CT absorption bands ascribed to different mutual donor/acceptor orientations favoring the overlap of the acceptor LUMO with either the HOMO or HOMO-1 of the donor. ${ }^{22,23}$ Excitation wavelength-dependent transient absorption studies of CR in DACs have been rationalized in terms of non-equilibrium CR in a mixture of tightly and loosely bound complexes. ${ }^{24,25}$ Braun and Sutin explained variations in free-ion yields following excitation at different wavelengths by invoking an equilibrium between well-defined DACs and 'random' solvent-separated pairs with a distribution of DA separations. ${ }^{26,27}$ Vibrational wavepackets observed in ultrafast studies of DACs in 
liquids were originally ascribed to intermolecular modes of the complexes, ${ }^{28,29}$ similar to those measured below $\sim 100 \mathrm{~cm}^{-1}$ in crystalline DACs. ${ }^{30}$ However, subsequent investigations revealed that these wavepackets originated from vibrational modes localized on either D or A subunits. ${ }^{31-33}$ It was concluded that the absence of intermolecular vibrational wavepackets most probably reflects a distribution of DAC geometries in the ground state. Finally, polarized transient electronic absorption measurements revealed significantly lower anisotropy values than those expected for perfectly formed $\pi$ stacked 'sandwich' structures. ${ }^{25,29,34,35}$ These low values were interpreted in terms of either multiple distinct geometries or structural disorder, but no detailed modeling of the anisotropies was carried out.

The present work attempts to address three primary questions regarding the structure and dynamics of DACs:

1. What structures do DACs adopt in the ground state? Are they predominately face-to-face $\pi$-stacks? How much heterogeneity is present?

2. Of these ground-state structures, which can undergo CT excitation? These excitable neutral pairs will determine the structure of the ion pairs at the onset of the CR process.

3. How does the excited-state population evolve after excitation? Do the IPs keep the same structure from which they were excited, or do they adopt a different conformation or set of conformations than in the ground state?

To this end, we have combined transient visible absorption (visTA) and polarized time-resolved infrared absorption (TRIR) spectroscopies with mixed quantum/classical computer simulations. We have chosen to focus on the well-known pair of benzene (Bz, donor) and tetracyanoethylene (TCNE, acceptor) solvated in $\mathrm{CH}_{2} \mathrm{Cl}_{2}$, which has been the subject of recent experimental $^{36,37}$ and computational investigations. ${ }^{38,39}$ The Bz/TCNE pair has a single CT absorption band peaked near $400 \mathrm{~nm}$, allowing for easy excitation using the doubled fundamental of a Ti:Sapphire laser system, and exhibits sufficiently slow ( $\sim 50 \mathrm{ps}$ ) CR to enable observation of the complete excited state structural dynamics. Additionally, the TCNE radical anion, $\mathrm{TCNE}^{\bullet-}$, has strong vibrational markers associated with the $\mathrm{C} \equiv \mathrm{N}$ stretching modes. ${ }^{40}$ These vibrational transition are located around $2150 \mathrm{~cm}^{-1}$, a relatively uncongested region of the IR spectrum, and have proven useful in previous infrared studies of DACs and bimolecular electron transfer using TCNE as an acceptor. ${ }^{41,42}$

We employ both visTA and TRIR measurements in order to provide an overview of the excited-state population dynamics. Polarized TRIR is then used to measure time-resolved vibrational anisotropy and obtain unprecedented insight into the geometry of excited DACs and their structural dynamics. To support the experiments, we have undertaken a systematic computational study of the $\mathrm{Bz} / \mathrm{TCNE} / \mathrm{CH}_{2} \mathrm{Cl}_{2}$ system combining quantum chemical calculations at the density-functional level of theory (DFT) and classical molecular dynamics (MD) simulations. Using umbrella sampling techniques and time-dependent density functional theory (TD-DFT) calculations we are able to characterize the ground- and excited-state distribution of Bz/TCNE structures as well as identify a distribution of ground-state geometries that allow for CT excitation. We then perform non-equilibrium MD in order to model the experimental TRIR anisotropy dynamics with help from the TD-DFT calculations. After validation by comparison to the experimental visible absorption lineshape and TRIR anisotropy, the results of the non-equilibrium MD simulations are used to examine translational diffusion of the ion pairs. The results of this study reveal that the Bz/TCNE DAC cannot be thought of as a well-defined ground-state complex that undergoes CT excitation to a static ion-pair structure. The anisotropy experiments and computations point to an initial population of structurally disordered ion pairs that relax to a predominantly T-shaped arrangement and not a $\pi$-stack as is usually assumed. Given the demonstrated sensitivity of charge-transfer processes to donor/acceptor orientation, ${ }^{43,44}$ theoretical descriptions of charge recombination dynamics in DACs must take into account DA pair structural diversity and the evolution of the excited state in order to properly model the charge recombination reaction.

\section{Methods}

\subsection{Materials}

HPLC grade benzene, toluene, $p$-xylene, and $\mathrm{CH}_{2} \mathrm{Cl}_{2}$ were purchased from Sigma-Aldrich and used as received. Tetracyanoethylene was purchased from Sigma-Aldrich and sublimed before use. Samples were prepared by adding donor to 0.04 M TCNE in $\mathrm{CH}_{2} \mathrm{Cl}_{2}$ to reach an absorbance at $400 \mathrm{~nm}$ of 0.1 in the $1 \mathrm{~mm}$ visTA cell and $0.2-0.3$ in the $0.3 \mathrm{~mm}$ TRIR cell. Given the small association constant of the binary complexes ${ }^{45}$ and the low donor and acceptor concentrations we have employed, we expect negligible amounts of the ternary complex to be present. The steady-state UV-vis absorption spectra of the visTA and TRIR samples were identical despite the differences in benzene concentration.

\subsection{Spectroscopy}

Steady-state UV-vis absorption measurements were performed using a Cary 50 spectrophotometer. Previously described instruments were used for the visible transient absorption (visTA), ${ }^{46,47}$ and infrared transient absorption (TRIR) measurements. ${ }^{48,49}$ Instrumental and data processing details are provided in Sections S1 and $\mathrm{S} 2$ of the ESI. +

\subsection{Computations}

All quantum chemical calculations were carried out using the Gaussian09 Rev. D.01 package $^{50}$ with the CAM-B3LYP functional ${ }^{51}$ and a $6-31 \mathrm{G}(\mathrm{d}, \mathrm{p})$ basis set. Electrostatic potentials generated from these calculations were fit using the CHELPG method ${ }^{52}$ to assign atomic charges to $\mathrm{Bz}, \mathrm{Bz}^{\bullet+}, \mathrm{TCNE}$, and $\mathrm{TCNE}^{\bullet-}$ for use as solutes in the MD simulations. Linear response TD-DFT calculations using the same functional and basis set were performed on select Bz/TCNE conformations in order to calculate the 5 lowest energy electronic transitions of the pairs. Molecular orbitals were visualized using the Avogadro 1.2.0 software package. ${ }^{53}$

All molecular dynamics (MD) simulations were performed using the GROMACS 2018.1 package, ${ }^{54}$ and the OPLS-AA force field. ${ }^{55}$ 
Simulation parameters and procedural details for equilibration, umbrella sampling, potential of mean force, and simulated TRIR anisotropy calculations are provided in Sections S3-S5 of the ESI.+ The MD trajectories were visualized using the VMD 1.9.3 package $^{56}$ and the Tachyon ray tracing library. ${ }^{57}$

\section{Results and discussion}

\subsection{Electronic transient absorption}

The steady-state electronic absorption spectrum of Bz/TCNE in $\mathrm{CH}_{2} \mathrm{Cl}_{2}$ consists of a single broad, featureless, CT band with a maximum at $388 \mathrm{~nm}$ (Fig. S1, ESI ). Neither Bz nor TCNE absorb in this region, meaning that the excited-state population upon $400 \mathrm{~nm}$ excitation originates solely from CT transitions of Bz/TCNE. Representative visTA spectra of Bz/TCNE in $\mathrm{CH}_{2} \mathrm{Cl}_{2}$ are presented in the top panel of Fig. 1. Although these data are generally poor in structural information, they provide a detailed

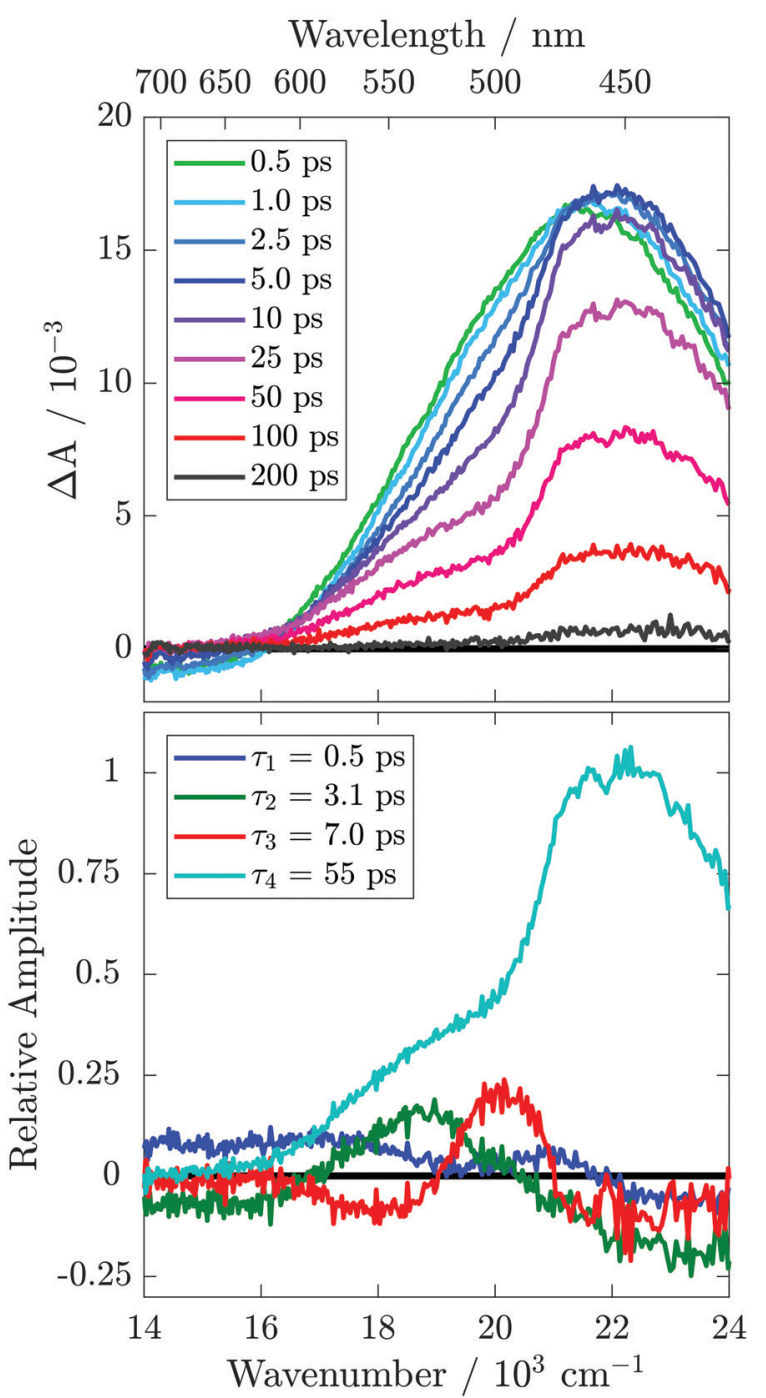

Fig. 1 Top: VisTA spectra of benzene/TCNE in $\mathrm{CH}_{2} \mathrm{Cl}_{2}$ following $400 \mathrm{~nm}$ excitation. Bottom: DADS from a four exponential global analysis of the visTA data in the top panel. view of the excited-state population dynamics. VisTA spectra collected at early times consist of a weak negative band below $16500 \mathrm{~cm}^{-1}(606 \mathrm{~nm})$, and a broad positive band peaking at $22000 \mathrm{~cm}^{-1}(455 \mathrm{~nm})$. During the first $10 \mathrm{ps}$ this broad feature undergoes a narrowing on its low-energy side, whereas the negative band decays to zero. At later times, the relaxed positive band decays entirely within $\sim 200$ ps.

Decay-associated difference spectra (DADS) and time constants resulting from a global multiexponential analysis of the visTA spectra are presented in the bottom panel of Fig. 1. Corresponding evolution-associated difference spectra (EADS), obtained by assuming successive exponential steps in the global analysis, are presented in Fig. S2 of the ESI. $\$$ A sum of four exponential functions is required to reproduce the spectra consisting of three components with sub-10 ps time constants that appear in the DADS as both rises and decays with a complicated wavelength dependence. A fourth component is present with a 55 ps time constant that is always a decay, and its DADS is nearly identical to the long-time visTA spectrum. The negative band coincides well with the steady-state fluorescence spectrum of this DAC reported by Chiu et $a .^{36}$ and can be assigned to stimulated emission. Its sub-10 ps decay together with the concomitant narrowing on the low-energy side of the positive band are interpreted as relaxation from the emissive Franck-Condon state to the equilibrium, IP-like, excited-state of the DAC. This is consistent with the electronic absorption spectra of $\mathrm{TCNE}^{\bullet-}$ and $\mathrm{Bz}^{\bullet+}$ reported in literature. ${ }^{58-60}$ Equilibration of the Franck-Condon state should involve vibrational, structural and solvent relaxation processes which cannot be disentangled, as they occur on similar timescales. Structural relaxation results in a decrease of the electronic coupling between the $\mathrm{D}$ and $\mathrm{A}$ subunits, hence the rapid decay of the stimulated emission. The complexity of the early dynamics is reflected by the need for three exponential functions to reproduce it. On the other hand, the 55 ps component can be attributed to the $\mathrm{CR}$ of the $\mathrm{Bz}^{\bullet+} / \mathrm{TCNE}^{\bullet-}$ ion pairs. The visTA spectra do not substantially decrease in intensity before $10 \mathrm{ps}$, suggesting that no significant CR occurs before equilibration of the excited DAC. Our recombination time of 55 ps is somewhat slower than the 29 ps reported by Chiu et al. ${ }^{36}$ using timeresolved fluorescence. This experiment is only able to examine the recombination of fluorescent pairs, whereas our visTA measurements include contributions from all pairs, possibly explaining the discrepancy in the recombination times. The 55 ps time constant is consistent with values reported for other DACs with a similar energy gap for recombination in medium polarity solvents. ${ }^{25,61,62}$

\subsection{Time-resolved IR absorption and anisotropy}

3.2.1 Population dynamics. In order to learn more about the structural dynamics of the excited DAC, we turn to timeresolved infrared absorption spectroscopy (TRIR). In these experiments, we pump the CT transition with an ultrashort $400 \mathrm{~nm}$ pulse and probe the symmetric and anti-symmetric $\mathrm{C} \equiv \mathrm{N}$ stretching modes of $\mathrm{TCNE}^{\bullet-}$ centered near $2150 \mathrm{~cm}^{-1}$ and $2185 \mathrm{~cm}^{-1}$. ${ }^{40}$ Such spectra of Bz/TCNE in $\mathrm{CH}_{2} \mathrm{Cl}_{2}$ are 


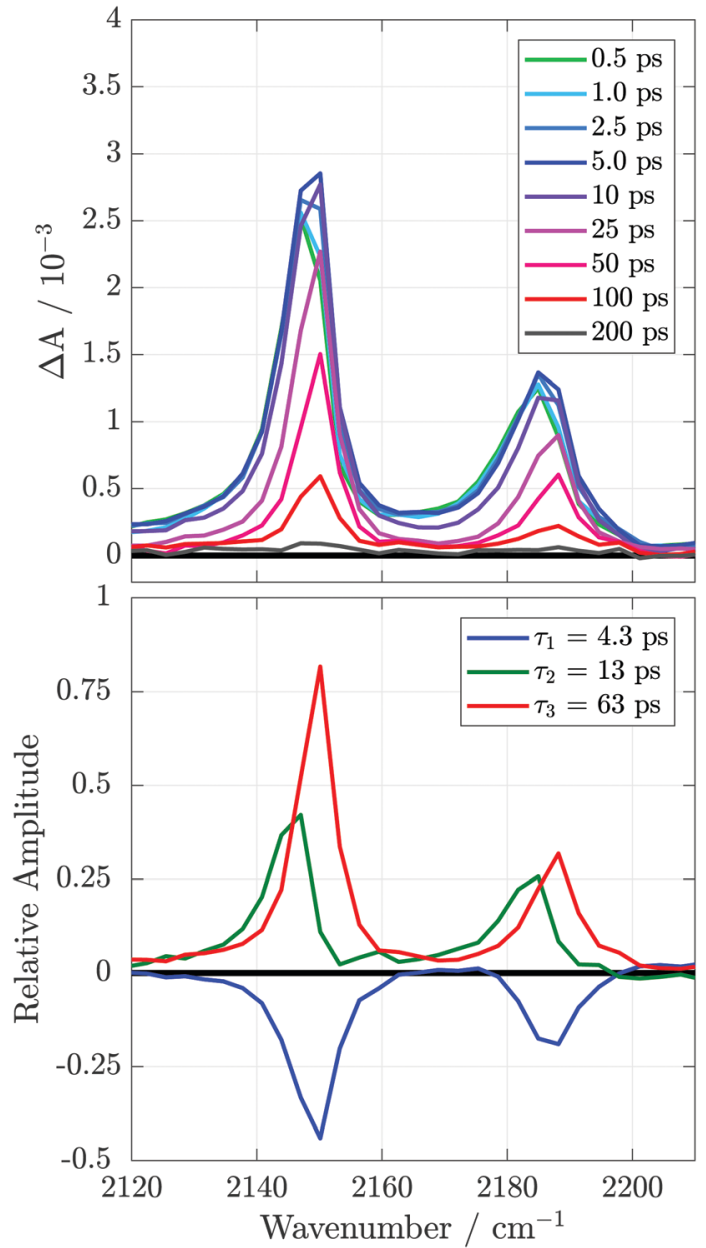

Fig. 2 Top: TRIR spectra of benzene/TCNE in $\mathrm{CH}_{2} \mathrm{Cl}_{2}$ in the nitrile stretch region following $400 \mathrm{~nm}$ excitation. Bottom: DADS from a three exponential global analysis of TRIR data in the top panel.

presented the top panel of Fig. 2. These data show that both $\mathrm{C} \equiv \mathrm{N}$ stretching bands undergo a frequency upshift of $\sim 4 \mathrm{~cm}^{-1}$ and a slight $(\sim 10 \%)$ increase in intensity before decaying. Similar dynamics have been explained in previous TRIR studies of bimolecular ET processes involving TCNE as indicative of tight (lower frequency) and loose (higher frequency) ion pairs, but were not observed in direct CT excitation due to prohibitively fast recombination. ${ }^{42}$ Blue shifts and narrowing in TRIR spectra are also observed in vibrational cooling and energy redistribution processes. ${ }^{63,64}$ Given the relatively large amount of excess energy deposited into the pair following $400 \mathrm{~nm}$ excitation, these processes are also expected to contribute to the spectral dynamics.

Three exponential components were sufficient for reproducing the TRIR dynamics using a global analysis. The resulting time constants and DADS are presented in the bottom panel of Fig. 2 and EADS in Fig. S3 (ESI $\$$ ). The initial rise and frequency up-shift are associated with the 4.3 and 13 ps time constants whereas the decay occurs with a 63 ps time constant. These data are consistent with the visTA and draw a similar picture of the excited state population dynamics. The DAC Franck-Condon

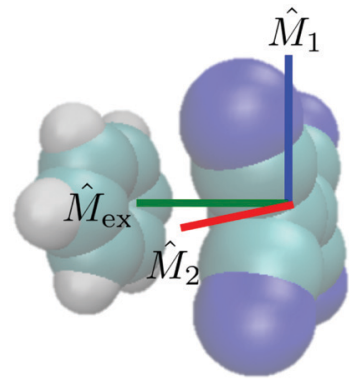

Fig. 3 Schematic representation of the Bz/TCNE DAC with experimentally relevant visible and vibrational transition dipole moments labeled. The vector $\hat{M}_{\text {ex }}$ represents the pumped CT transition dipole moment, and $\hat{M}_{1}$ and $\hat{M}_{2}$ represent the nitrile vibrations probed in $\operatorname{TCNE}^{\bullet}$.

state equilibrates on a $\sim 10$ ps time scale through vibrational, solvent, and conformational relaxation, and the ensuing ion pair recombines with a $\sim 60$ ps time constant. Unfortunately, the TRIR population dynamics themselves do not yield significant structural information about the pairs.

3.2.2 TRIR anisotropy. Unlike standard absorption measurements, anisotropy experiments provide information on the relative orientation of the transition dipole moments involved in the pump and probe processes. Specifically, TRIR anisotropy reports on the orientation of the pumped electronic transition dipole moment, $\hat{M}_{\mathrm{ex}}$, relative to the probed IR transition moment $\hat{M}_{i}$ (outlined in Fig. 3), according to ${ }^{65}$

$$
r^{(i)}(t)=\frac{2}{5}\left(\frac{3}{2}\left\langle\hat{M}_{\mathrm{ex}}(0) \cdot \hat{M}_{i}(t)\right\rangle^{2}-\frac{1}{2}\right), \quad i=1,2 .
$$

The term in brackets is the second rank rotational correlation function of $\hat{M}_{\text {ex }}$ and $\hat{M}_{i}$, providing a direct link to the excitedstate structure and dynamics.

Before presenting the anisotropy data, we must first describe the transition dipole moments involved in the TRIR measurements and the anticipated geometry of the Bz/TCNE pair. Fig. 3 illustrates a canonical $\pi$-stacked 'sandwich' DAC with the orientations of the electronic and vibrational transition dipole moments labeled. The electronic CT transition dipole of DACs is known to lie perpendicular to the molecular planes of the donor and acceptor, whereas the probed $\mathrm{C} \equiv \mathrm{N}$ vibrational transition dipole moments both lie in the molecular plane of TCNE and are mutually perpendicular. ${ }^{42}$ We define $\hat{M}_{1}$ as the transition dipole moment of the $2150 \mathrm{~cm}^{-1}$ antisymmetric stretch which lies along the $\mathrm{C}=\mathrm{C}$ bond and $\hat{M}_{2}$ as the transition dipole moment of the symmetric stretch near $2185 \mathrm{~cm}^{-1}$ which lies in the TCNE molecular plane orthogonal to $\hat{M}_{1}$.

The TRIR anisotropy of Bz/TCNE measured at 2147 and $2188 \mathrm{~cm}^{-1}$ is presented in Fig. 4 and best-fit parameters in Table 1. Population and anisotropy time constants from fits at different frequencies of the TRIR spectrum are provided in Table S1 of the ESI. $\$$ We observe a clearly bimodal anisotropy at $2147 \mathrm{~cm}^{-1}$ with a 5.9 ps rise and a 47 ps decay, whereas the $2188 \mathrm{~cm}^{-1}$ anisotropy begins negative and simply relaxes to zero on the order of 10 ps. The decay of the negative anisotropy of both bands occurs on a similar timescale as the spectral dynamics in 


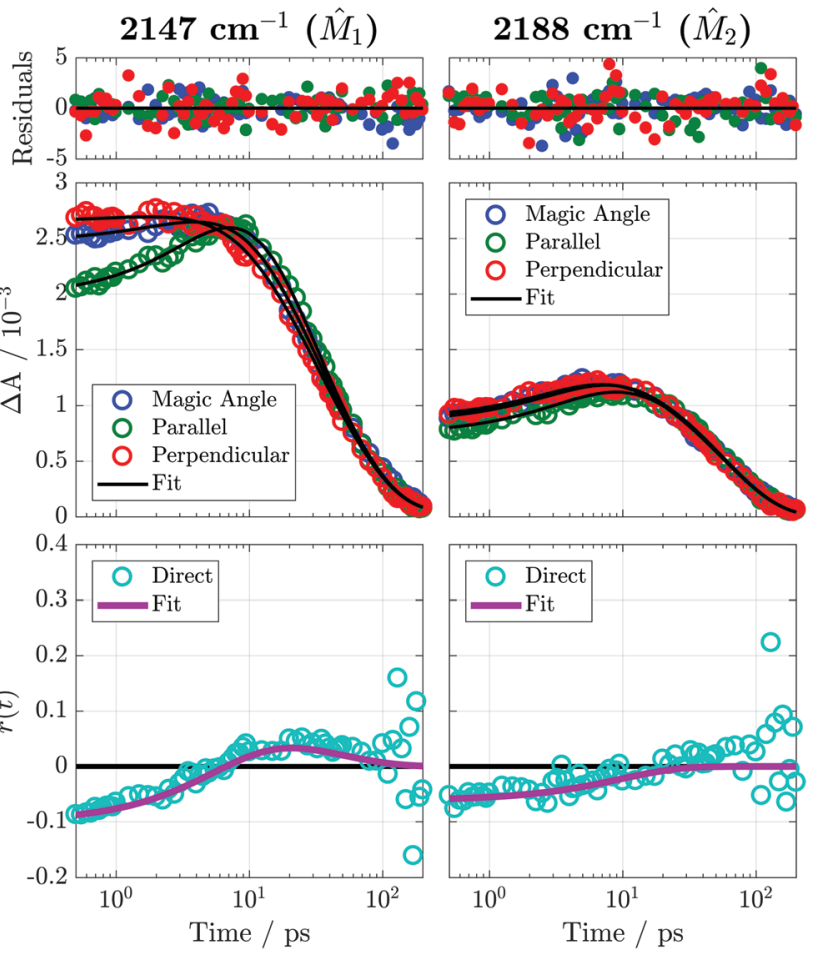

Fig. 4 Polarized TRIR time traces and anisotropy measured with $\mathrm{Bz} /$ TCNE $/ \mathrm{CH}_{2} \mathrm{Cl}_{2}$ at $2147 \mathrm{~cm}^{-1}$ and $2188 \mathrm{~cm}^{-1}$ following $400 \mathrm{~nm}$ excitation. Residuals were weighted using the standard error of the 8 individual measurements as uncertainties. These uncertainties would be approximately the size of the symbols if plotted as error bars. Fitting of the TRIR traces and the anisotropy was performed using the model described in Section S2 of the ESI.\$ The points labeled 'direct' are the anisotropy calculated using the definition $r(t)=\left[\Delta A_{\|}(t)-\Delta A_{\perp}(t)\right] /\left[\Delta A_{\|}(t)+2 \Delta A_{\perp}(t)\right]$. This representation of the anisotropy was not analyzed directly but is intended to serve as a check on the fitting procedure.

Table 1 Best-fit parameters extracted from the TRIR anisotropy data presented in Fig. 4 using the model described in Section S2 of the ESI. Uncertainties of $10-30 \%$ in these parameters are estimated based on the standard deviation of replicate measurements. The largest uncertainty is found in the slow anisotropy time constant $\tau_{r, 2}$, as it is on the order of the slowest population time constant, $\tau_{3}$

Population dynamics

\begin{tabular}{lllllllll}
\hline$\tilde{\nu}\left(\mathrm{cm}^{-1}\right)$ & $h$ & $b k g$ & $a_{1}$ & $\tau_{1}(\mathrm{ps})$ & $a_{2}$ & $\tau_{2}(\mathrm{ps})$ & $a_{3}$ & $\tau_{3}(\mathrm{ps})$ \\
\hline 2147 & 4.77 & 0.04 & -0.25 & 4.9 & 0.26 & 15 & 0.50 & 50 \\
2188 & 2.29 & 0.02 & -0.14 & 2.1 & -0.18 & 8.6 & 0.68 & 51 \\
\hline
\end{tabular}

Anisotropy dynamics

\begin{tabular}{lllllll}
\hline$\tilde{\nu}\left(\mathrm{cm}^{-1}\right)$ & $G$ & $r_{0}$ & $r_{1}$ & $\tau_{\mathrm{r}, 1}(\mathrm{ps})$ & $r_{2}$ & $\tau_{\mathrm{r}, 2}(\mathrm{ps})$ \\
\hline 2147 & 0.976 & -0.10 & -0.16 & 5.9 & 0.06 & 47 \\
2188 & 0.979 & -0.06 & -0.06 & 9.8 & - & -
\end{tabular}

the visTA and TRIR spectra, possibly reflecting a shared physical origin. The best-fit parameters in Table S1 (ESI exhibit a significant frequency dependence within a band and the variation most likely reflects uncertainties in the data.

A number of processes could be responsible for the temporal evolution of the anisotropy including: (1) changes in the mutual orientation of the D and A subunits, (2) reorientation of the whole excited complex, and (3) decay of subpopulations of excited DACs with a well-defined geometry and faster CR dynamics. As discussed earlier, no significant population decay occurs during the first 10 ps and, consequently, process (3) should not be important here. The Stokes-Einstein-Debye (SED) prediction for the reorientation time of the whole complex is 19.4 ps using a van der Waals volume of $194 \AA^{3},{ }^{66}$ a $\mathrm{CH}_{2} \mathrm{Cl}_{2}$ viscosity of $0.411 \mathrm{mPa}$ s at $298 \mathrm{~K},{ }^{67}$ and stick boundary conditions. This is significantly longer than the $\tau_{\mathrm{r}, 1}$ values measured here. Time constants on the order of SED predictions were observed in earlier TRIR anisotropy studies of exciplexes of 9-cyanoanthracene and phthalic anhydride in acetonitrile and tetrahydrofuran. ${ }^{48}$ Therefore, the decay of the negative anisotropy can be attributed to structural relaxation of the DAC after excitation and the slow decay of the positive anisotropy found with $\hat{M}_{1}$ to the reorientation of the complex and randomization of Bz/TCNE orientations.

A qualitative interpretation of the initial anisotropies can be attempted using the schematic in Fig. 3 as a guide. If all excited DACs were sandwich structures as depicted in Fig. 3, we would expect an initial anisotropy of $r_{0}^{(i)}=-0.2$ for both probed vibrations and not the values of $r_{0}^{(1)}=-0.10$ and $r_{0}^{(2)}=-0.06$ seen in the experiment. Other sandwich structures obtained by rotating TCNE about the $\hat{M}_{\text {ex }}$ axis would have the same initial anisotropy of -0.2 . Therefore, the reduced initial anisotropies must result from rotation of the Bz and TCNE molecular planes with respect to each other. The observed values of $r_{0}^{(i)}$ could be the result of (1) significant orientational disorder, (2) multiple well-defined structures with different anisotropies, or (3) a single structure with an angle of $65^{\circ}$ between $\hat{M}_{\text {ex }}$ and $\hat{M}_{1}$ and $61^{\circ}$ between $\hat{M}_{\text {ex }}$ and $\hat{M}_{2}$. Because the anisotropy experiment reports only on ensemble averaged orientations, one cannot discriminate between these three cases without resorting to detailed modeling of the system. The sign change from negative to positive in the $2147 \mathrm{~cm}^{-1}$ anisotropy indicates that $\hat{M}_{1}$ initially rotates into the same direction as $\hat{M}_{\text {ex }}$, whereas $\hat{M}_{2}$ either randomizes or adopts a magic angle $\left(54.7^{\circ}\right)$ orientation with respect to $\hat{M}_{\text {ex }}$. These dynamics are consistent with the formation of edge-to-face (T-shaped) or edge-to-edge structures, but we do not have the benzene-specific anisotropies required to discriminate between the two, as the TRIR bands of $\mathrm{Bz}^{\circ+}$ modes were too weak to reliably extract anisotropies.

It should be noted that these anisotropies are not unique to the Bz/TCNE complex. TRIR anisotropy measurements of toluene/TCNE and $p$-xylene/TCNE complexes in $\mathrm{CH}_{2} \mathrm{Cl}_{2}$, presented in Fig. S4, S5 and Tables S2, S3 (ESI $\$$ ), exhibit somewhat stronger initial anisotropies with $r_{0}$ ranging from -0.14 to -0.17 with single exponential relaxation that appears slower than in Bz/TCNE. Unfortunately, charge recombination in these pairs is 5-10 times faster than in Bz/TCNE due to different driving forces for these reactions, quickly depopulating the excited state and obscuring the long-time ( $>10 \mathrm{ps})$ anisotropy. Therefore, we cannot be confident in the anisotropy time constants and cannot determine if the $\hat{M}_{1}$ anisotropy changes sign, an unambiguous indicator of structural rearrangement. 
Regardless of the lack of long time data, the larger initial anisotropies and clearly slower anisotropy dynamics may be due to the formation or preferential excitation of tighter face-to-face complexes. Further interpretation of the experimental anisotropies is difficult, as there is no unique distribution of structures that can reproduce the observed anisotropy; the experiments only report on the ensemble average of structures and their corresponding anisotropies. In order to learn more about the structures and dynamics reported on by the anisotropy we must turn to computer simulations validated by comparison to the experiments.

\subsection{Mixed quantum/classical computations}

A combined quantum/classical computational study of the Bz/ TCNE system was undertaken in order to aid interpretation of the TRIR anisotropies. There are a number of key physical processes which must be modeled in order to simulate the experimental anisotropy in a sufficiently rigorous manner. First, we must determine which $\mathrm{Bz} / \mathrm{TCNE}$ structures are present in the ground state and which of these are able to undergo CT excitation. Then we must simulate the dynamics of the ion pairs resulting from the CT excitation and calculate anisotropies according to eqn (1). To accomplish this, we combine classical MD simulations with TD-DFT calculations using the following procedure:

1. Characterize the ground-state $\mathrm{Bz} / \mathrm{TCNE}$ structures using the potential of mean force calculated from umbrella sampling MD simulations.

2. Select a representative set of ground-state Bz/TCNE conformations from the umbrella sampling simulations and use TD-DFT to calculate CT transition energies, transition dipole moments, and oscillator strengths.

3. Using the same set of configurations, run non-equilibrium MD trajectories after substituting Bz/TCNE charges with those of $\mathrm{Bz}^{\bullet+} / \mathrm{TCNE}^{\bullet-}$. This serves to model the CT transition as an instantaneous transfer of a full electron from Bz to TCNE.

4. Calculate an anisotropy for each non-equilibrium trajectory according to eqn (1) using CT transition dipole moments from the TD-DFT calculations for $\hat{M}_{\mathrm{ex}}(0)$ and $\mathrm{TCNE}^{\bullet-}$ coordinates from the simulations to calculate $\hat{M}_{1}(t)$ and $\hat{M}_{2}(t)$. The final simulated anisotropy is calculated by averaging the individual trajectory anisotropies weighted by the CT transition oscillator strength at $t=0$.

3.3.1 Potentials of mean force and conformation selection. We begin by simulating the potential of mean force (PMF) between neutral $\mathrm{Bz}$ and TCNE as a function of center-of-mass separation, $w(r)$, using umbrella sampling techniques (see Section S3 for computational details, ESI $\$)$. This function characterizes the free energy of interaction between two moieties as a function of separation distance and is related to the pair distribution function by $g(r)=\exp \left[-w(r) / k_{\mathrm{B}} T\right] .{ }^{68}$ We additionally simulated $w(r)$ for the ion pair in order to gain insight into the expected excited state equilibrium. The results of these simulations are presented in Fig. 5.

The neutral pair $w(r)$ has a minimum of $-0.3 k_{\mathrm{B}} T$ at $r=0.37 \mathrm{~nm}$ $\left(-0.66 \mathrm{~kJ} \mathrm{~mol}^{-1}\right.$ at $\left.T=293.15 \mathrm{~K}\right)$. Because this free energy is significantly smaller than $k_{\mathrm{B}} T$, we conclude there is little attraction between neutral $\mathrm{Bz}$ and TCNE in room temperature $\mathrm{CH}_{2} \mathrm{Cl}_{2}$.

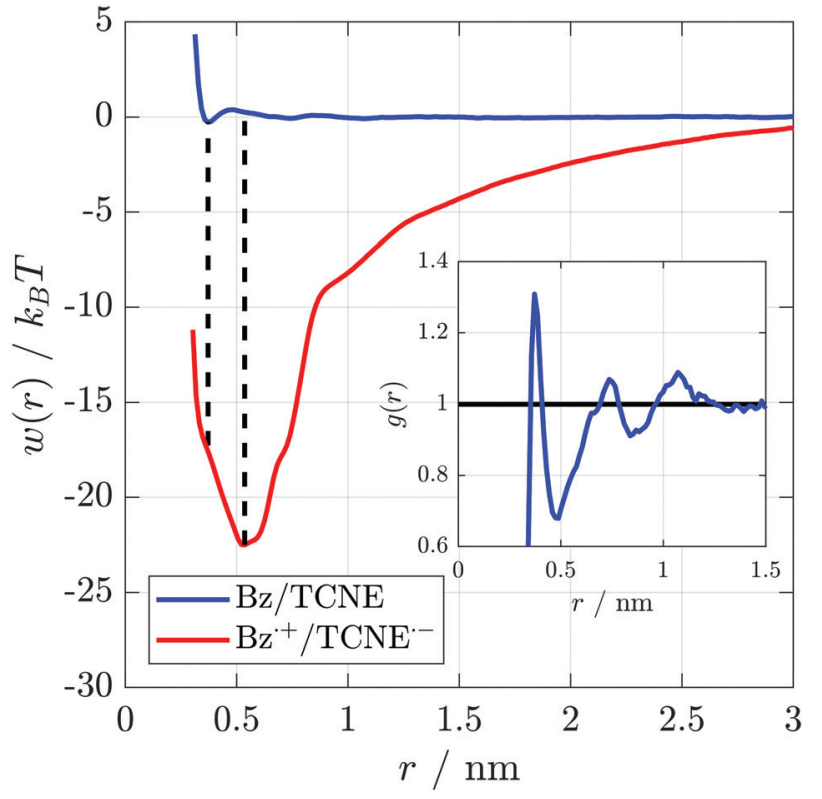

Fig. 5 Potentials of mean force (PMFs) for neutral Bz/TCNE and ionic $\mathrm{Bz}^{\bullet+} / \mathrm{TCNE}^{\bullet-}$ pairs. The black dashed lines are intended to guide the eye toward the minima of the neutral and ion pair PMFs. Inset: Neutral Bz/ TCNE pair distribution function used for selection of conformations for the TD-DFT calculations and non-equilibrium MD simulations.

Rather than as ground state complexes, it is better to consider $\mathrm{Bz} / \mathrm{TCNE}$ pairs in this solvent as dilute cosolvents. The shallow $w(r)$ reported here is consistent with the weak association constant of $0.1 \mathrm{~L} \mathrm{~mol}^{-1}$ measured for Bz/TCNE complexes using steady-state UV-vis absorption. ${ }^{45}$ In contrast to the neutrals, the ion pair $w(r)$ has a significantly deeper minimum of $-22.5 k_{\mathrm{B}} T$ and is found at a longer separation, $r=0.54 \mathrm{~nm}$. The difference in the location of the minimum and the large change in interaction strength point to significant structural rearrangement following excitation. The initially disordered (in $r$ ) ground-state population will be funneled into the deep and narrow minimum of the ionic $w(r)$ after excitation, effecting a significant conformational change. The depth of this minimum also suggests that no free ions will form on an experimentally significant timescale. We expect that the high degree of structural diversity demonstrated in the Bz/TCNE DAC studied here to be present in other DACs as well. Preliminary PMF calculations for pairs of dimethylaniline and aromatic acceptors in acetonitrile (Fig. S6, ESI ) exhibit similarly shallow minima and would be expected to have structural disorder similar to that of Bz/TCNE.

We turn to orientational distribution functions, $G(\theta)$, to characterize mutual Bz/TCNE orientations of the neutral and ionic pairs. After selection of appropriate molecular vectors, these distributions are calculated from Bz/TCNE conformations generated by the PMF calculations according to:

$$
G(\theta)=\langle\delta(\theta-\phi)\rangle
$$

where $\phi$ is the angle between selected $\mathrm{Bz}$ and TCNE molecular vectors. Fig. 6 shows the results of such calculations for in-plane (IP) and one out-of-plane (OOP) Bz/TCNE and $\mathrm{Bz}^{\bullet+} / \mathrm{TCNE}^{\bullet-}$ molecular vectors for pairs with $r$ within $0.025 \mathrm{~nm}$ of their 




Neutral

lonic
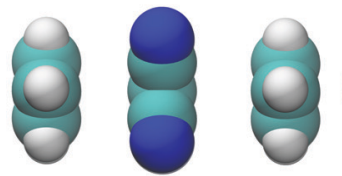

Fig. 6 Orientational distributions for Bz and TCNE pairs within $0.025 \mathrm{~nm}$ of the minimum of $w(r)$ for the neutral (blue) and ionic (red) species. The angles $\theta_{\mathrm{OOP}}$, and $\theta_{\mathrm{IP}}$ represent the angles between the out-of-plane and in-plane molecular vectors indicated on each plot. These distributions are normalized for unit area. The gray lines represent the expectation for an isotropic distribution of orientations. Conformations depicted beneath the plots represent the most probable Bz/TCNE structures predicted by the orientational distributions.

respective $w(r)$ minimum. The in-plane vector of TCNE is selected to lie along its short axis (the same as $\hat{M}_{1}$ in Fig. 3) and for $\mathrm{Bz}$ originates at its center-of-mass and points between two $\mathrm{Bz} \mathrm{H}$ atoms. The out-of-plane vectors are simply normal to the $\mathrm{Bz}$ and TCNE molecular planes. These vectors are illustrated by the inset images in Fig. 6 . Note that these distributions are by definition weighted by $\sin (\theta)$, therefore, an isotropic distribution of orientations corresponds to a sin function as illustrated by the gray lines in Fig. 6. A similar approach was recently used to predict the relative orientation of the individual constituents in perylene diimide aggregates. ${ }^{69}$

The out-of-plane vectors of neutral Bz/TCNE show a strong preference for mutual orientations of 0 and $\pi$, as would be expected for a face-to-face sandwich structure. There are much weaker preferences for orientations of 0 and $\pi$ for the in-plane angle. Together, these distributions allow us to conclude that the neutral pair $w(r)$ minimum does indeed correspond to a sandwich structure, but this geometry is not strongly preferred due to the weak attraction between neutral $\mathrm{Bz}$ and TCNE exemplified by the shallow PMF. On the other hand, the ion pairs assume a significantly different structure than their neutral counter parts. Both out-of-plane and in-plane vectors now show strong preferences for orientations of $\pi / 2$. These distributions suggest that the dominant ion pair structure is edge-to-face or T-shaped, with the long-edge of $\mathrm{TCNE}^{\bullet-}$ rotated toward the benzene molecular plane. By taking the difference of the ion pair PMF at the neutral and ion pair PMF minima, we estimate a driving force of $\sim-5 k_{\mathrm{B}} T$ for the formation of the T-shaped pair. Illustrations of the conformations predicted for the neutral and ion pair are depicted at the bottom of Fig. 6 . These structures are roughly consistent with our experimentally observed anisotropies. The sandwich structures predicted by the neutral $w(r)$ and expected to be most easily excited are associated with a negative anisotropy for both $\hat{M}_{1}$ and $\hat{M}_{2}$ at short times, and diffusion into T-shaped structures is consistent with the change of sign observed in the $\hat{M}_{1}$ anisotropy and the lack of sign change for $\hat{M}_{2}$.

3.3.2 Quantum-chemical calculations. Now that we have characterized the equilibrium structures of neutral and ionic $\mathrm{Bz} / \mathrm{TCNE}$, we must determine if the structures generated by the PMF calculations are a realistic representation of the Bz/TCNE ground state and, if so, to test if they can be used to model the excited state ion pair dynamics using non-equilibrium MD. To do this, we start by using the pair distribution function of the neutral pair $(g(r)$, inset of Fig. 5$)$ to select $\sim 1500$ representative ground state $\mathrm{Bz} / \mathrm{TCNE}$ conformations for the TD-DFT calculations and non-equilibrium MD simulations. Details of the conformation selection procedure are provided in Section S4 of the ESI. $\neq$

We begin by modeling the experimental steady-state absorption lineshape, $A(\tilde{\nu}) / \tilde{\nu}$, using the results of TD-DFT calculations on the representative Bz/TCNE conformations selected from the PMF simulations. Bz/TCNE coordinates are extracted from each selected conformation, all solvent molecules stripped away, and a TD-DFT calculation is performed on the remaining $\mathrm{Bz} / \mathrm{TCNE}$ atoms to determine the electronic transition energies, transition dipole moments, and oscillator strengths for the 5 lowest energy transitions. We find that the energy of the first two transitions ranges from 18000 to nearly $32000 \mathrm{~cm}^{-1}$ and corresponds primarily to one-electron excitations from the HOMO and HOMO-1 of Bz to the LUMO of TCNE. The higher transitions, which are more than $2500 \mathrm{~cm}^{-1}$ higher in energy, correspond primarily to local excitations of Bz or TCNE, and lie far outside the CT band observed in experiment. Oscillator strengths and transition frequencies for all transitions are presented in Fig. S7 and an example Bz/TCNE conformation and frontier molecular orbitals involved in the first two transitions are given in Fig. S8 (ESI ). All further analyses will only concern the two lowest energy transitions which will be referred to hereafter as the 'CT transitions'.

The oscillator strengths for the CT transitions are plotted against their transition energy in the top panel of Fig. 7. Despite the two-fold degeneracy of the isolated benzene HOMO, the first two transitions form two slightly separated bands. This is a 


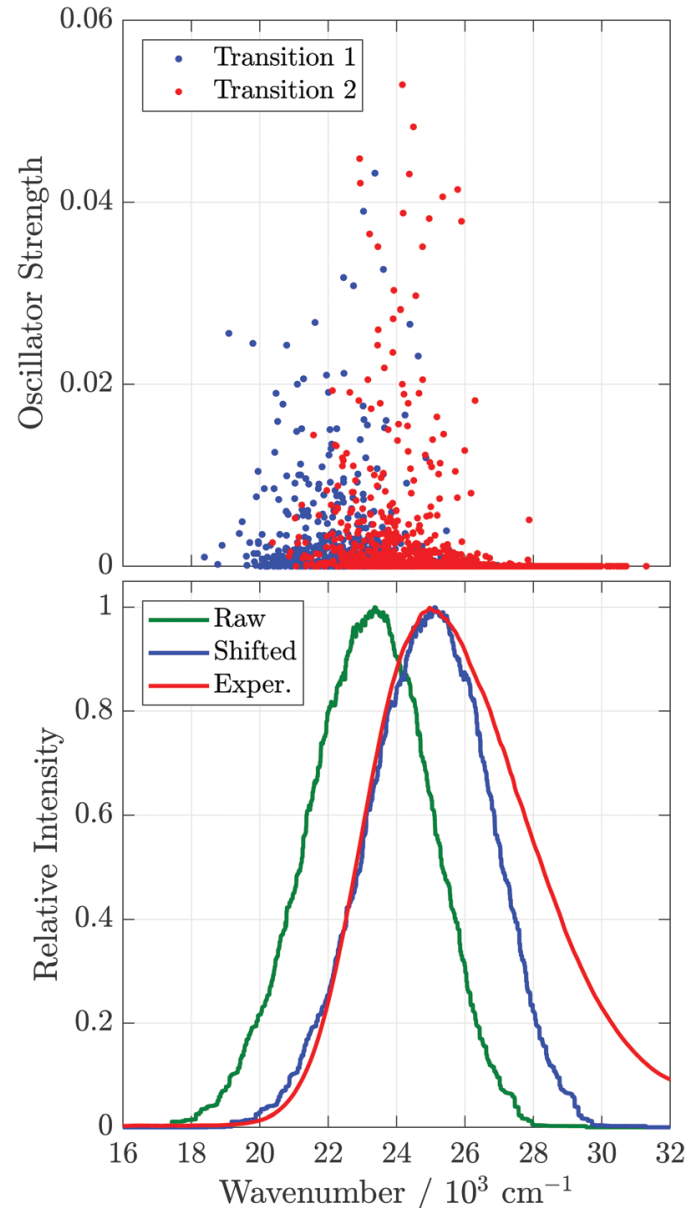

Fig. 7 Top: Oscillator strengths of the two Bz/TCNE CT transitions vs. transition energy. Bottom: Experimental and simulated Bz/TCNE/C $\mathrm{H}_{2} \mathrm{Cl}_{2}$ absorption lineshapes. The green curve labeled 'Raw' is the result of binning and smoothing the oscillator strengths in the top panel, the blue curve labeled 'Shifted' is the 'Raw' curve up-shifted by $1750 \mathrm{~cm}^{-1}$, and the red curve labeled 'Exper.' is the experimental absorption lineshape, $A(\tilde{\nu}) / \tilde{\nu}$.

consequence of the benzene molecular orbital symmetry and the mutual orientation of benzene and TCNE. Although, in isolation, the benzene HOMO is two-fold degenerate, the two HOMOs differ spatially, primarily by rotation of $90^{\circ}$ about the $\mathrm{Bz} C_{6}$ symmetry axis (Fig. S9, ESI\$). Consequently, benzene HOMO energies (and therefore CT transition frequencies) will split depending on the relative orientation of benzene and TCNE. This phenomenon is analogous to the split bands observed in the absorption spectra of substituted benzene DACs. $^{22,23}$ In these DACs, the degeneracy of the HOMO is broken due to asymmetrical substitution and two distinct absorption bands are observed, corresponding to CT from either the HOMO or HOMO-1 of the donor to the LUMO of TCNE. In the case of benzene, the difference in transition energy is due to differences in donor and acceptor MO overlap and not differences in the isolated donor HOMO energies. This splitting is much weaker than that induced by substitution, and therefore the absorption spectrum appears to be a single broad band.
We approximate the measured absorption lineshape by binning the CT transition oscillator strengths by transition energy and smooth the resulting histogram using a movingaverage filter. The results of such calculations and the experimental absorption lineshape are presented in the bottom plot of Fig. 7. The raw simulated lineshape must be up-shifted by $1750 \mathrm{~cm}^{-1}(0.22 \mathrm{eV})$ in order to match the maximum of the experimental lineshape. This is not unexpected, as the TD-DFT calculations are performed in vacuum and do not include the solvent environment, either explicitly or through continuum models. After shifting, the calculated spectra appear to reproduce the red-edge of the absorption spectrum, but do not properly capture the asymmetry of the band at high frequencies, possibly due to the lack of vibronic progression ${ }^{7,9}$ and solvent environment in the TD-DFT calculations. Despite these omissions, the width of the experimental absorption lineshape $\left(\mathrm{FWHM}=5500 \mathrm{~cm}^{-1}\right)$ is captured reasonably well by the simulation $\left(\mathrm{FWHM}=4200 \mathrm{~cm}^{-1}\right.$ ), suggesting that the broadening of the experimental lineshape can be well accounted for by Bz/TCNE structural heterogeneity. In previous simulations, which included vibronic transitions but not inhomogeneous broadening due to structural heterogeneity, unexpectedly large reorganization energies had to be assumed to properly reproduce the CT lineshape of DACs in apolar solvents. ${ }^{28,70}$ The good agreement obtained here suggests the $\mathrm{MD}$ generated $\mathrm{Bz} / \mathrm{TCNE}$ pairs are a reasonable representation of the $\mathrm{Bz} / \mathrm{TCNE}$ ground state.

Before moving on to the non-equilibrium simulations, we pause to consider the oscillator strength and transition energy dependence on separation distance as depicted in Fig. 8 . Although pairs separated by less than $0.40 \mathrm{~nm}$ (approximately the first minimum in the neutral pair $g(r)$ ) exhibit the largest CT oscillator strengths, a significant number of these short-range pairs have weak or no oscillator strength. Concomitantly, there are pairs at separations up to $0.75 \mathrm{~nm}$ with significant oscillator strength, both supporting the $r$ cutoff used in the conformation selection procedure and confirming that long-distance pairs will contribute to the excited-state population at $t=0$. Calculated transition energies, $\tilde{\nu}_{\mathrm{CT}}^{(i)}$, are plotted as a function of separation in the bottom panel of Fig. 8. We see that both CT transition energies vary greatly even for the same $r$, with a spread of $\sim 4000 \mathrm{~cm}^{-1}$, due to the fact that the same $r$ may be associated with a number of distinct geometries. Consequently, $r$ alone is clearly not a sufficient descriptor of the DAC structure. Smoothing these data with a moving-average filter (green and yellow curves, bottom panel Fig. 8) allow us to more easily follow how the mean excitation energy changes with $r$. The two transition energies are approximately constant at 22000 and $23600 \mathrm{~cm}^{-1}$ for $r<0.55 \mathrm{~nm}$ and increase monotonically to 26200 and $28500 \mathrm{~cm}^{-1}$ at $r=0.91 \mathrm{~nm}$. Although these smoothed curves might appear to suggest the magnitude of transition energy splitting is constant, the splitting can range from 400 to $5000 \mathrm{~cm}^{-1}$ and has no correlation with $r$ (Fig. S10, ESI ). Therefore, the splitting is a sensitive function of $\mathrm{Bz} / \mathrm{TCNE}$ mutual orientation and not separation distance.

3.3.3 Non-equilibrium MD and simulated anisotropy. The conformations chosen for the TD-DFT calculations were used 



Fig. 8 Distance dependence of the CT oscillator strengths (top) and transition energies (bottom) obtained from TD-DFT calculations, where $r$ is the center-of-mass separation of the Bz/TCNE pair. The smoothed transition energies in the bottom panel were calculated using a moving average filter. A frequency shift was not applied to these data.

as starting points for non-equilibrium MD simulations in order to simulate the ion pair dynamics following excitation. For each configuration, the charges of neutral $\mathrm{Bz}$ and TCNE were swapped for those of their radical ions and the system propagated for $1 \mathrm{~ns}$. The simulated TRIR anisotropy was then calculated using $\mathrm{TCNE}^{\bullet-}$ orientations generated by the simulations in conjunction with transition dipole moments and oscillator strengths from the TDDFT calculations. Details of the anisotropy calculation are provided in Section S5 of the ESI. $\$$ Simulated anisotropies and their experimental counterparts are presented in Fig. 9 and parameters of exponential fits of the simulated anisotropy are given in Table 2 . Despite the relative simplicity of the modeling, the simulated anisotropies strikingly reproduce the major features of the experiment. The simulated $\hat{M}_{1}$ anisotropy begins negative and changes sign within a few picoseconds, whereas $\hat{M}_{2}$ starts slightly less negative than $\hat{M}_{1}$ and relaxes to 0 on a timescale similar to the experiment. Initial anisotropies are reproduced nearly exactly and further demonstrate the realism of the ground state previously described by the PMF and TD-DFT calculations.

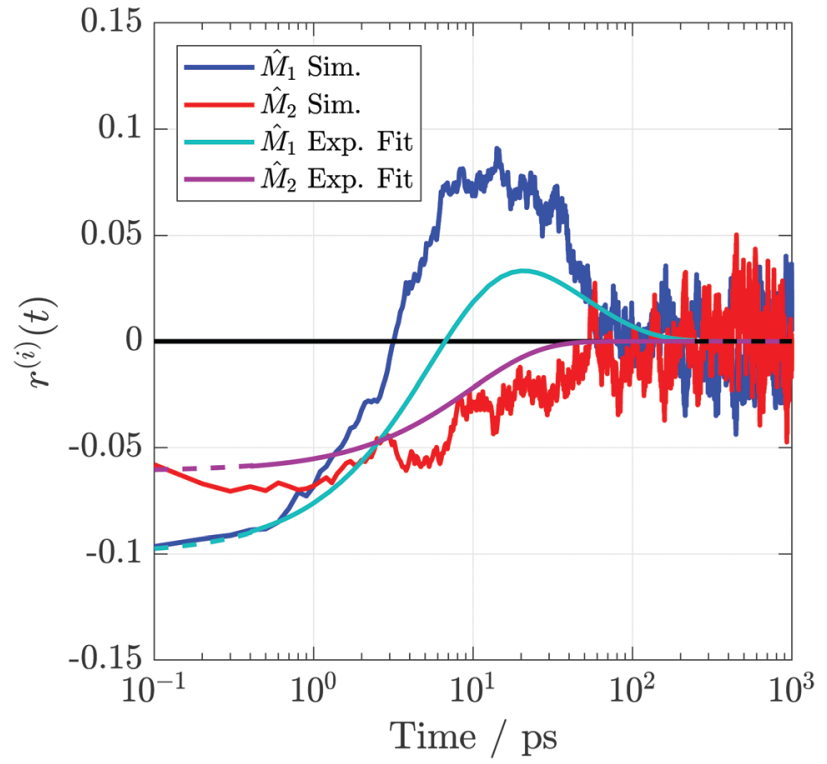

Fig. 9 TRIR anisotropies from the simulations and fits to the experimental data. Axes definitions are as outlined in Fig. 3. Dashed portions of the experimental curves indicate extrapolations outside the collected temporal region.

Table 2 Best-fit parameters from exponential fits to the simulated anisotropies in Fig. 9

\begin{tabular}{llllll}
\hline Vector & $r_{0}$ & $r_{1}$ & $\tau_{\mathrm{r}, 1}(\mathrm{ps})$ & $r_{2}$ & $\tau_{\mathrm{r}, 2}(\mathrm{ps})$ \\
\hline$\hat{M}_{1}$ & -0.11 & -0.28 & 5.2 & 0.17 & 28 \\
$\hat{M}_{2}$ & -0.07 & -0.07 & 20 & - & -
\end{tabular}

The apparent success in reproducing the experimental anisotropy allows us to be confident in using the non-equilibrium simulations to study the excited state structural dynamics in greater detail. Oscillator strength weighted orientational distributions calculated at $t=0$ and $t=1$ ns (Fig. S11, ESI, $\$$ analogous to those in Fig. 6 but with orientations weighted by the $t=0$ oscillator strength), are consistent with those predicted by the PMF calculations, but with more disorder. The disorder in these distributions compared to those calculated from the PMFs comes from considering all selected initial conformations, ranging from 0.31 to $0.95 \mathrm{~nm}$, instead of only focusing on the minimum in the PMF as was done in Fig. 6. These data confirm that the structural rearrangement predicted by the equilibrium PMF calculations does in fact take place.

To attempt to visualize the Bz/TCNE 'chromophore', we have created composite images by overlaying Bz/TCNE conformations at $t=0$ and 1 ns from each non-equilibrium MD trajectory. The transparency (alpha channel) of each individual image was weighted by the conformation's $t=0$ oscillator strength in order to approximate its contribution to the absorption lineshape/excited state population. The resulting images are presented in Fig. 10, and TCNE carbon atoms have been removed for clarity. The $t=0$ image shows that although excitation of Bz/TCNE comes predominately from face-to-face structures, other, less ordered, conformations contribute significantly to the excitation. This disorder is the source of 


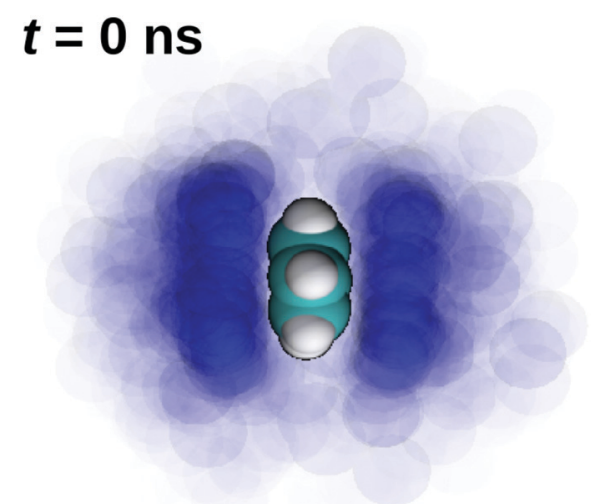

$t=1 \mathrm{~ns}$

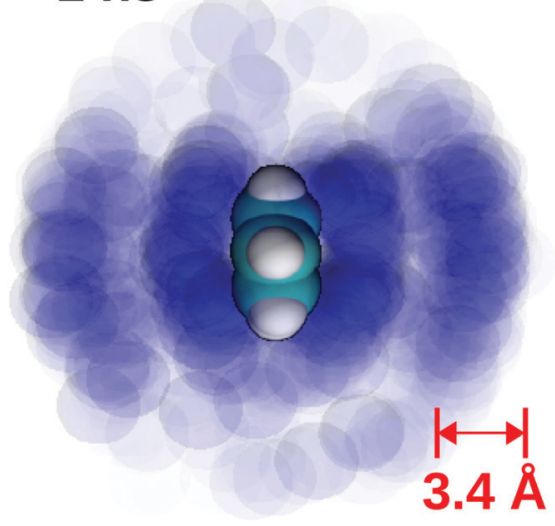

Fig. 10 An approximation of the Bz/TCNE 'chromophore' created by superimposing an image of each Bz/TCNE conformation from nonequilibrium MD simulations at $t=0$ and $1 \mathrm{~ns}$. The blue spheres represent TCNE nitrogen atoms and the TCNE carbon atoms have been omitted for clarity. The transparency of each individual image is weighted by the conformation's $t=0$ oscillator strength in order to approximate its contribution to the excited state population.

the reduced initial anisotropy seen in the TRIR anisotropy experiments. At $t=1 \mathrm{~ns}$ the ion pairs have reached a conformational equilibrium where a majority of the $\mathrm{Bz}^{\bullet+} / \mathrm{TCNE}^{\bullet-}$ pairs are oriented in an edge-to-face configuration. Despite the strong Coulombic attraction between the ions, the pairs are still considerably disordered. These images clearly demonstrate the stark structural differences between neutral and ion pair DACs and their accompanying heterogeneity; this DAC cannot be described as a single static species.

In order to follow translational diffusion of the ion pairs, we examine the time-dependent distributions of center-of-mass separation, $p(r ; t)$, as presented in Fig. 11. These calculations are related to the time-dependent pair distribution function, $g(r ; t)$, but are normalized simply for unit area instead of the bulk density. Therefore, $p(r ; t)$ represents the evolution of the ion-pair center-of-mass distribution from $t=0$, determined by the neutral pair $w(r)$ to their equilibrium distribution, determined by the ion pair $w(r)$ (blue and red curves, Fig. 5 ). We note that these distributions will not be weighted by oscillator strength in order to highlight the pure ion pair dynamics.

The top panel of Fig. 11 illustrates the short-time evolution of $p(r ; t)$. At $t=0$, we see a peak near $0.37 \mathrm{~nm}$ that corresponds to
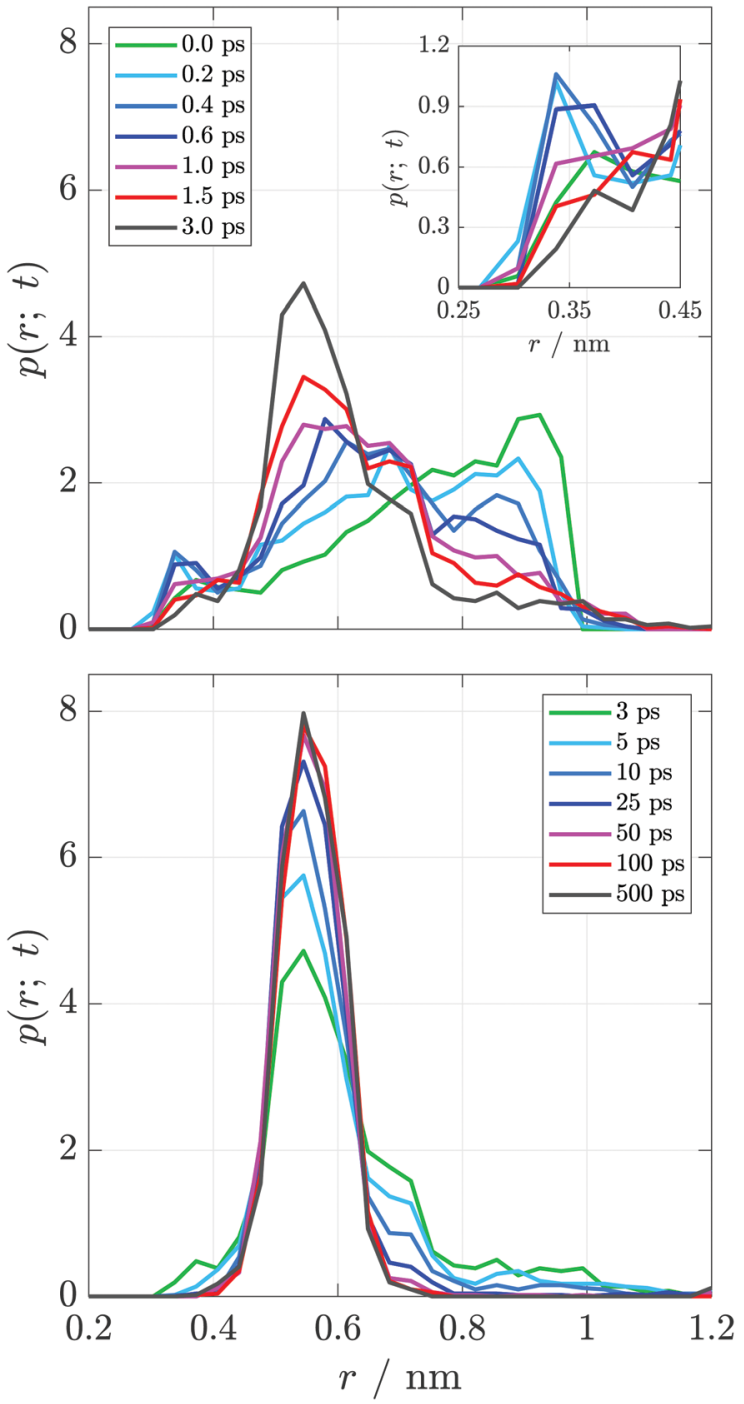

Fig. 11 Time-dependent distributions of center-of-mass separation from the non-equilibrium MD simulations.

the minimum in $w(r)$ of the neutral pairs. This peak shifts in $0.2 \mathrm{ps}$ to $0.34 \mathrm{~nm}$ before relaxing towards the ion pair $w(r)$ minimum of $0.54 \mathrm{~nm}$ with a small side peak at $r \approx 0.40 \mathrm{~nm}$. From the long side, $r>0.70 \mathrm{~nm}$, the distribution contracts towards the ion-pair minimum, but a secondary shoulder also appears at $r \approx 0.7 \mathrm{~nm}$. The long-time evolution of $p(r ; t)$ in the bottom panel of Fig. 11 shows that the two side peaks seen in the short time distributions eventually collapse into the ionpair minimum. The short side of the distribution collapses faster as evidenced by the faster rise on the short side than the long side.

These dynamics suggest that $w(r)$ is not sufficient by itself to properly describe the translational dynamics of the photogenerated ions. There is only a single minimum in $w(r)$, and the derivative of $w(r)$ is always negative for $r<r_{\min }$ and positive for $r>r_{\min }$ (Fig. S12, ESI $\$$ ) indicating that there are no local minima in $w(r)$ and therefore no metastable states for the ion pairs to sample (e.g. the short time contraction feature or side 
peaks in the long-time $p(r ; t))$. We can conclude that the structural relaxation in this system must happen on a multidimensional potential surface that includes both separation and orientation coordinates. This is also supported by the starkly different orientational distributions for the neutral and ionic species shown in Fig. 6. The path a particular ion pair follows to reach the ion pair equilibrium position will then depend on its initial position on this multidimensional surface. Multidimensional diffusion involving separation and solvent coordinates has been used to describe the ion pairing mechanism of monovalent ion pairs in water, ${ }^{71,72}$ and the diffusion seen in the molecular ions here can be seen as an extension of these dynamics involving additional orientational coordinates.

\section{Conclusions}

The experiments and simulations herein paint a significantly different portrait of the DAC ground and excited-state in liquids than is typically assumed by the single structure $\pi$-stacked 'sandwich' model. The excited-state dynamics of the Bz/TCNE DAC begin with a $\sim 10$ ps equilibration involving vibrational, solvent and structural relaxation from the initial FranckCondon state to an ion-pair-like state, as evidenced by the complicated spectral dynamics observed in both visTA and TRIR measurements. Spectral relaxation is followed by the charge recombination of the ion pairs with a time-constant of 55-60 ps. TRIR anisotropy experiments reveal starkly different dynamics for two different $\mathrm{TCNE}^{\bullet-}$ vibrations and indicate structural reorganization of the ion pairs following excitation.

Using a combination of TD-DFT calculations and molecular dynamics simulations, we were able to satisfactorily reproduce the experimental steady-state visible absorption spectrum and TRIR anisotropy. The results of these calculations demonstrate that even though a $\pi$-stack is the most probable ground state structure, the attraction between neutral Bz/TCNE in $\mathrm{CH}_{2} \mathrm{Cl}_{2}$ is less than $k_{\mathrm{B}} T$ and a high degree of disorder is present. On the other hand, $\mathrm{Bz}^{\bullet+} / \mathrm{TCNE}^{\bullet-}$ ion pairs preferentially adopt edge-toface $\mathrm{T}$-shaped structures and not the $\pi$-stack as usually assumed. This result suggests that the ground-state structure of the DAC is primarily determined by dispersion interactions, whereas that of the ion-pair like excited state is governed by Coulombic interactions. The TD-DFT calculations on representative MD generated $\mathrm{Bz} / \mathrm{TCNE}$ structures revealed a strong sensitivity of the CT transition oscillator strength and transition energy to the mutual Bz/TCNE orientation and a weak dependence on separation. Therefore, optical excitation in the CT band results in a photoselection of geometries with a significant oscillator strength. However, these excitable pairs still form a highly disordered ensemble with disparate centerto-center distances and D/A orientations illustrated in the composite images of Fig. 10. The apparent success in reproducing the visible absorption lineshape using vacuum phase TD-DFT calculations suggests that broadening in experimental spectrum is dominated by structural inhomogeneity. Nonequilibrium $\mathrm{MD}$ simulations of the $\mathrm{Bz}^{\bullet+} / \mathrm{TCNE}^{\bullet-}$ pair exhibit dynamics inconsistent with diffusion on a one dimensional potential energy surface and indicates the presence of coupled rotational-translational diffusion and heterogeneous structural dynamics. Further simulations and quantum-chemical calculations on this system and other pairs are needed in order to understand the interplay between pair structure and solvent properties on the DAC absorption spectrum, excited-state orientational and translational dynamics, and charge recombination.

\section{Conflicts of interest}

There are no conflicts to declare.

\section{Acknowledgements}

We thank Joseph S. Beckwith for assistance in collecting the visTA data and Alexander Zech for providing the CCParser Python module used for extracting data from the TD-DFT calculations. The quantum chemical and molecular dynamics computations were performed at University of Geneva on the Baobab cluster. We are also grateful for the financial support provided by the Swiss National Science Foundation (project no. 200020-165890) and the University of Geneva.

\section{References}

1 R. S. Mulliken, J. Am. Chem. Soc., 1952, 74, 811-824.

2 E. F. Hilinski, J. M. Masnovi, J. K. Kochi and P. M. Rentzepis, J. Am. Chem. Soc., 1984, 106, 8071-8077.

3 N. Mataga and H. Miyasaka, Adv. Chem. Phys., 1999, 107, 431-496.

4 J. M. Masnovi and J. K. Kochi, J. Am. Chem. Soc., 1985, 107, 7880-7893.

5 T. Asahi and N. Mataga, J. Phys. Chem., 1989, 93, 6575-6578.

6 T. Kumpulainen, B. Lang, A. Rosspeintner and E. Vauthey, Chem. Rev., 2017, 117, 10826-10939.

7 F. Markel, N. S. Ferris, I. R. Gould and A. B. Myers, J. Am. Chem. Soc., 1992, 114, 6208-6219.

8 A. B. Myers, Chem. Phys., 1994, 180, 215.

9 M. Lilichenko, D. Tittelbach-Helmrich, J. W. Verhoeven, I. R. Gould and A. B. Myers, J. Chem. Phys., 1998, 109, 10958-10969.

10 O. Nicolet and E. Vauthey, J. Phys. Chem. A, 2002, 106, 5553-5562.

11 R. G. Fedunov, S. V. Feskov, A. I. Ivanov, O. Nicolet, S. Pagès and E. Vauthey, J. Chem. Phys., 2004, 121, 3643-3656.

12 S. V. Feskov, V. A. Mikhailova and A. I. Ivanov, J. Photochem. Photobiol., C, 2016, 29, 48-72.

13 E. Adman, M. Rosenblum, S. Sullivan and T. N. Margulis, J. Am. Chem. Soc., 1967, 89, 4540-4542.

14 F. K. Larsen, R. G. Little and P. Coppens, Acta Crystallogr., Sect. B: Struct. Crystallogr. Cryst. Chem., 1975, 31, 430-440.

15 F. A. Balmer, P. Ottiger, C. Pfaffen and S. Leutwyler, J. Phys. Chem. A, 2013, 117, 10702-10713.

16 F. A. Balmer, P. Ottiger and S. Leutwyler, J. Phys. Chem. A, 2015, 119, 10462-10474. 
17 M. Hayashi, T.-S. Yang, J. Yu, A. Mebel and S. H. Lin, J. Phys. Chem. A, 1997, 101, 4156-4162.

18 M.-S. Liao, Y. Lu, V. D. Parker and S. Scheiner, J. Phys. Chem. A, 2003, 107, 8939-8948.

19 J. A. Ciezak, J. B. Leão and B. S. Hudson, THEOCHEM, 2006, 767, 23-28.

20 D. Kuchenbecker and G. Jansen, ChemPhysChem, 2012, 13, 2769-2776.

21 T. S. Kolesnikova, M. S. Chernov'yants, M. E. Kletskii, O. N. Burov, G. I. Bondarenko and P. A. Knyazev, J. Phys. Chem. A, 2017, 121, 7000-7008.

22 E.-M. Voigt, J. Am. Chem. Soc., 1964, 86, 3611-3617.

23 M. Rossi, U. Buser and E. Haselbach, Helv. Chim. Acta, 1976, 59, 1039-1053.

24 O. Nicolet, N. Banerji, S. Pagès and E. Vauthey, J. Phys. Chem. A, 2005, 109, 8236-8245.

25 O. F. Mohammed and E. Vauthey, J. Phys. Chem. A, 2008, 112, 5804-5809.

26 J. Zhou, B. R. Findley, A. Teslja, C. L. Braun and N. Sutin, J. Phys. Chem. A, 2000, 104, 11512-11521.

27 J. Zhou, B. R. Findley, C. L. Braun and N. Sutin, J. Chem. Phys., 2001, 114, 10448-10456.

28 K. Wynne, C. Galli and R. M. Hochstrasser, J. Chem. Phys., 1994, 100, 4797-4810.

29 K. Wynne, G. D. Reid and R. M. Hochstrasser, J. Chem. Phys., 1996, 105, 2287-2297.

30 M. Rossi and E. Haselbach, Helv. Chim. Acta, 1979, 62, 140-151.

31 I. V. Rubtsov and K. Yoshihara, J. Phys. Chem. A, 1997, 101, 6138-6140.

32 M. Hayashi, T.-S. Yang, J. Yu, A. Mebel, R. Chang, S. H. Lin, I. V. Rubtsov and K. Yoshihara, J. Phys. Chem. A, 1998, 102, 4256-4265.

33 I. V. Rubtsov and K. Yoshihara, J. Phys. Chem. A, 1999, 103, 10202-10212.

34 B. R. Arnold, A. W. Schill and P. V. Poliakov, J. Phys. Chem. A, 2001, 105, 537-543.

35 B. R. Arnold, A. Euler, P. V. Poliakov and A. W. Schill, J. Phys. Chem. A, 2001, 105, 10404-10412.

36 C.-C. Chiu, C.-C. Hung, C.-L. Chen and P.-Y. Cheng, J. Phys. Chem. B, 2013, 117, 9734-9756.

37 C.-C. Chiu, C.-C. Hung and P.-Y. Cheng, J. Phys. Chem. B, 2016, 120, 12390-12403.

38 G. Piacente, A. Amadei, M. D’Abramo, I. Daidone and M. Aschi, Phys. Chem. Chem. Phys., 2014, 16, 20624-20638.

39 A. A. J. Aquino, I. Borges, R. Nieman, A. Köhn and H. Lischka, Phys. Chem. Chem. Phys., 2014, 16, 20586-20597.

40 J. S. Miller, Angew. Chem., Int. Ed., 2006, 45, 2508-2525.

41 O. F. Mohammed, N. Banerji, B. Lang, E. T. J. Nibbering and E. Vauthey, J. Phys. Chem. A, 2006, 110, 13676-13680.

42 O. F. Mohammed, K. Adamczyk, N. Banerji, J. Dreyer, B. Lang, E. T. J. Nibbering and E. Vauthey, Angew. Chem., Int. Ed., 2008, 47, 9044-9048.

43 G. Angulo, A. Cuetos, A. Rosspeintner and E. Vauthey, J. Phys. Chem. A, 2013, 117, 8814-8825.

44 B. Wu, M. Maroncelli and E. W. Castner, J. Am. Chem. Soc., 2017, 139, 14568-14585.
45 J. E. Frey, A. M. Andrews, D. G. Ankoviac, D. N. Beaman, L. E. Du Pont, T. E. Elsner, S. R. Lang, M. A. O. Zwart, R. E. Seagle and L. A. Torreano, J. Org. Chem., 1990, 55, 606-624.

46 N. Banerji, G. Duvanel, A. Perez-Velasco, S. Maity, N. Sakai, S. Matile and E. Vauthey, J. Phys. Chem. A, 2009, 113, 8202-8212.

47 G. Duvanel, N. Banerji and E. Vauthey, J. Phys. Chem. A, 2007, 111, 5361-5369.

48 M. Koch, R. Letrun and E. Vauthey, J. Am. Chem. Soc., 2014, 136, 4066-4074.

49 B. Dereka, D. Svechkarev, A. Rosspeintner, M. Tromayer, R. Liska, A. M. Mohs and E. Vauthey, J. Am. Chem. Soc., 2017, 139, 16885-16893.

50 M. J. Frisch, G. W. Trucks, H. B. Schlegel, G. E. Scuseria, M. A. Robb, J. R. Cheeseman, G. Scalmani, V. Barone, B. Mennucci, G. A. Petersson, H. Nakatsuji, M. Caricato, X. Li, H. P. Hratchian, A. F. Izmaylov, J. Bloino, G. Zheng, J. L. Sonnenberg, M. Hada, M. Ehara, K. Toyota, R. Fukuda, J. Hasegawa, M. Ishida, T. Nakajima, Y. Honda, O. Kitao, H. Nakai, T. Vreven, J. A. Montgomery, J. E. Peralta, F. Ogliaro, M. Bearpark, J. J. Heyd, E. Brothers, K. N. Kudin, V. N. Staroverov, R. Kobayashi, J. Normand, K. Raghavachari, A. Rendell, J. C. Burant, S. S. Iyengar, J. Tomasi, M. Cossi, N. Rega, J. M. Millam, M. Klene, J. E. Knox, J. B. Cross, V. Bakken, C. Adamo, J. Jaramillo, R. Gomperts, R. E. Stratmann, O. Yazyev, A. J. Austin, R. Cammi, C. Pomelli, J. W. Ochterski, R. L. Martin, K. Morokuma, V. G. Zakrzewski, G. A. Voth, P. Salvador, J. J. Dannenberg, S. Dapprich, A. D. Daniels, O. Farkas, J. B. Foresman, J. V. Ortiz, J. Cioslowski and D. J. Fox, Gaussian 09, Revision D.01, Gaussian, Inc., Wallingford, CT, 2013.

51 T. Yanai, D. P. Tew and N. C. Handy, Chem. Phys. Lett., 2004, 393, 51-57.

52 L. E. Chirlian and M. M. Francl, J. Comput. Chem., 1987, 8, 894-905.

53 M. D. Hanwell, D. E. Curtis, D. C. Lonie, T. Vandermeersch, E. Zurek and G. R. Hutchison, J. Cheminform., 2012, 4, 17.

54 M. J. Abraham, T. Murtola, R. Schulz, S. Páll, J. C. Smith, B. Hess and E. Lindahl, SoftwareX, 2015, 1-2, 19-25.

55 W. L. Jorgensen, D. S. Maxwell and J. Tirado-Rives, J. Am. Chem. Soc., 1996, 118, 11225-11236.

56 W. Humphrey, A. Dalke and K. Schulten, J. Mol. Graphics, 1996, 14, 33-38.

57 J. Stone, MSc thesis, Computer Science Department, University of Missouri-Rolla, 1998.

58 T. Shida, Electronic Absorption Spectra of Radical Ions, Elsevier, Amsterdam, 1988, vol. 34.

59 Y. Kodera and T. Hikida, Spectrosc. Lett., 1989, 22, 1229-1239.

60 T. M. Bockman, Z. J. Karpinski, S. Sankararaman and J. K. Kochi, J. Am. Chem. Soc., 1992, 114, 1970.

61 T. Asahi, M. Ohkohchi and N. Mataga, J. Phys. Chem., 1993, 97, 13132-13137.

62 W. Jarzeba, S. Murata and M. Tachiya, Chem. Phys. Lett., 1999, 301, 347-355. 
63 P. Hamm, S. M. Ohline and W. Zinth, J. Chem. Phys., 1997, 106, 519-529.

64 M. Koch, A. Rosspeintner, K. Adamczyk, B. Lang, J. Dreyer, E. T. J. Nibbering and E. Vauthey, J. Am. Chem. Soc., 2013, 135, 9843-9848.

65 G. R. Fleming, Chemical Applications of Ultrafast Spectroscopy, Oxford University Press, 1986.

66 J. T. Edward, J. Chem. Educ., 1970, 47, 261.

67 Y. Marcus, The Properties of Solvents, Wiley, New York, 2005.
68 D. Chandler, Introduction to Modern Statistical Mechanics, Oxford University Press, New York, 1987.

69 M. A. Mattson, T. D. Green, P. T. Lake, M. McCullagh and A. T. Krummel, J. Phys. Chem. B, 2018, 122, 4891-4900.

70 K. Kulinowski, I. R. Gould and A. B. Myers, J. Phys. Chem., 1995, 99, 9017-9026.

71 S. Roy, M. D. Baer, C. J. Mundy and G. K. Schenter, J. Phys. Chem. C, 2016, 120, 7597-7605.

72 S. Roy, M. D. Baer, C. J. Mundy and G. K. Schenter, J. Chem. Theory Comput., 2017, 13, 3470-3477. 